\title{
Free Space Optics (FSO): A Promising Solution to First and Last Mile Connectivity (FLMC) in the Communication Networks
}

\author{
M Mubasher Hassan ${ }^{\mathrm{a}, \mathrm{b}^{*}}$, G M Rather \\ ${ }^{a}$ Research Scholar, ECE Department, National Institute of Technology (NIT), Srinagar, J\&K - 190006 \\ ${ }^{\mathrm{b}}$ Assistant Professor, BGSB University Rajouri (J\&K)-185234 \\ ${ }^{\mathrm{c}}$ Professor, Dept. of ECE, NIT Srinagar, J\&K - 190006 \\ Email: mubasher2003@gmail.com, gulammohdrather@yahoo.co.in
}

Received: 31 March 2020; Accepted: 02 May 2020; Published: 08 August 2020

\begin{abstract}
We are running in the communication era demanding a very large bandwidth for high speed applications. Among the available bandwidth technologies, the optical fiber seems the most appropriate and best fit. The optical fiber technology laid down at the backbone networks has almost replaced the preexisting coax cables. To extend fiber connectivity to the end user, especially in congested and remote areas is quite a difficult task in the context to cost and installation time. Therefore, the first and last mile connectivity (FLMC) is still a bottleneck in extending the beauty of optical fiber to the edge of the network. In most of the applications, the connectivity from backbone to the end user is carried out with the radio or copper links having a much lesser capacity than optical fiber. Keeping in view the nature and size of emerging applications, the FLMC needs to be addressed with the appropriate technology. To address this issue, the emerging solution is optical wireless communication like free space optics (FSO). The FSO is coming as a more promising alternative due to its properties like huge bandwidth and less cost. In this paper, we discuss the possible solution to first and last mile connectivity by FSO links and hence the gap between the optical core and the edge of network can be bridged by FSO communication in reliable and cost effective manner. The significance of the proposed work gives an everlasting impression of using FSO communication in FLMC over the existing ones. The ever increasing demand of high bandwidth can be meticulously met with the FSO communication. The simulation results show that the desirable performance is achieved and is analyzed with performance indicators like Q-factor and BER.
\end{abstract}

Index Terms: Free space optics, bandwidth requirement, optical wireless, first and last mile connectivity.

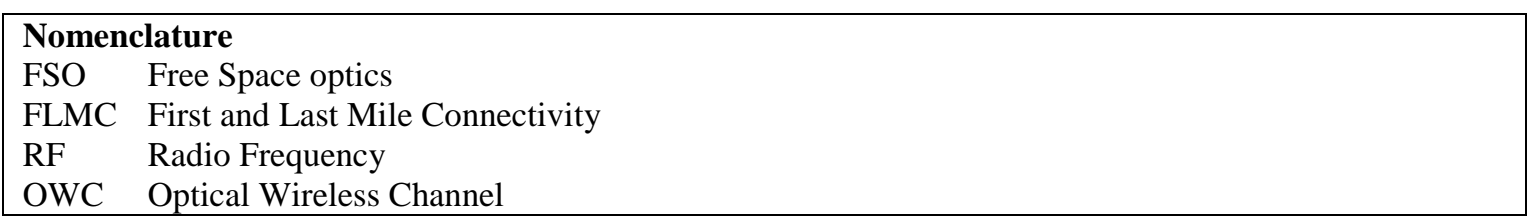

\section{Introduction}

The demand for high bandwidth in the present communication systems is increasing day by day. After every 18 months, the demand for bandwidth is doubled since the inception of data services and no slowing is noticed in this growth. The next generation internet connectivity will require infrastructure to cater high bandwidth applications such as video conferencing, streaming multimedia content and network enabled portable devices for every user. The exponential growth of electronic communication systems supporting a 
large number of high speed data applications depends on the available bandwidth technologies. We are heading towards all optical communication networks due to a very large available optical spectrum and establishing of optical fibre networks is carrying a substantial pace especially in the backbone networks. Keeping the merits of wireless communication under consideration, the connectivity at first and last end of the network is preferred wirelessly. This is presently being done using radio links, but the communication networks are demanding a very high bandwidth both at the core and at the edge of the network due to exponential increase in the telecommunication traffic. Due to the scarcity and lesser bandwidth of radio spectrum than optical spectrum, we are looking for the suitable alternative and one of the attractive solutions emerging is free space optics (FSO)[1]. Among the available alternative bandwidth technologies, FSO is considered to be the most suitable alternative due to the number of advantages like no licensing, easy to install, low cost, and high data rate. However, its capabilities are limited by local weather conditions like fog, rain, snow, haze and dust, etc. To reduce these attenuations is the topic of research and study. It looks promising that FSO communication can play a very pivotal role in the communication networks. It can bridge the gap, where optical fiber cable cannot be reached [2].

The adoption of a new technology is mostly based on some of the common parameters like cost effectiveness, time to market, re-configuration, reliability and maximum efficiency. Free space optical communication possesses all these features and hence best suitable for first and last mile connectivity. Presently the communication system should offer services with high speed and adequate security for all the data transmissions. Different types of information like voice, video, facsimiles and text etc has led to the development of multimedia signals and requires very high bandwidth for communication from one place to another. Optical wireless is most suitable to address this problem provided some limitations caused due to open atmosphere are suitability mitigated. FSO is a technology of transmitting data in the form of light through the atmosphere. The principle of operation is similar to the optical fibre communication, but characteristics of channel are totally different[3]. Here in FSO, the use of fibre cable is not required. The interface between transmitter and receiver in the FSO communication is air. Therefore, weather conditions need to be examined before the actual implementation of FSO link is established [4]. The possible solution to first and last mile connectivity by FSO is described in this paper. The demand of the future communication is a large capacity, better quality services, and sustainable broadband services. The bandwidth gap caused by legacy technologies between fiber on the one side and copper, and radio links on the other side has been the focus of attention at present. The paper aims to examine the supported evidence to the concept of using optical wireless links as an alternative to the radio links at FLMC. The non availability of high bandwidth in radio links as compared to optical fiber does not permit the seamless transmission of high data rate from the source to sink. The other objective of this study is to establish all optical connectivity between a transmitter and the receiver, as the optical signal possesses significant merits over the electric pulses in addition to the transmission capabilities. The proposed technique of WDM-NRZ modulation using FSO link is expected to deliver performance, which is desirable and acceptable.

\section{Brief Historical Background}

If we give a sharp look into the history of optical wireless communication, we find that the beginning of communication was started with light. The ancient people used to communicate nearby villages by burning fire and wanted to send them pre-coded information like some danger is occurring or death happened or any other information. The actual concept of FSO was taken into considerations in 8th century. The alphabetic system of signaling in the coded form was used by the ancient Greeks with torches developed by Polybius, Democleitus and Cleoxenus to inform their recipients. The first FSO photo-phone concept was practically demonstrated by Alexander Graham Bell, who is being recognized as pioneers of FSO communication. He transmitted voice wirelessly over the beam of light through the air, some $213 \mathrm{~m}$ apart in June 03, 1880 and dubbed this experiment as photo-phone. Though, this invention did not turn into commercial reality at that time. But after many decades the concept was transformed into reality in military communication telegraphy. During the world war-I, the wire communication was heavily suffered from frequent cuts. Three types of transmitters based on optical Morse called Blinkgerat were used by German 
signals for distance up to $4 \mathrm{~km}$ at day and up to $8 \mathrm{~km}$ at night. At the end of the war optical telephone communication was tested. Special Blinkgerats were used in balloons, airplanes and tanks for communication with varying success. Later on Carl Zeiss and Jena developed optical speaking devices and they were used in WW-II by German army in anti-aircraft defense units. In 1960, the FSO got revolutionized by the laser inventions. The FSO is emerging from niche to mainstream network infrastructure from past 15 years. The first vendor, namely Cable Free commercialized FSO with 62Mbps in 1997 and with 1Gbps in 1999. Due to adverse effects of atmospheric conditions, the growth of FSO communication was very much restrained and the corresponding need of wireless communication was addressed by RF technology at a greater degree of success. At present the scarcity in the RF spectrum puts a challenge before the communication scientists and engineers to search the alternative technology which is having very large bandwidth. Optical wireless like FSO is the best option having virtually unlimited unlicensed bandwidth. From last two decades FSO has attained significant attention in research and people are working in different areas of FSO technology to address the ill effects of the atmosphere. The FSO is evolving and also getting a substantial market value. Nowadays FSO is also being used at NASA to ensure very high speed wireless communication. After taking the stock of literature, it is imperative that FSO links can best be implemented at shorter distances and hence best suitable for first and last mile connectivity.

\section{Motivation towards Free Space Optics (FSO)}

At present the optical fibre is the most mature and reliable technology to address the bandwidth requirement in communication networks. But it has some inherent disadvantages like digging and which eventually requires permission from the people where from fibre is to be laid down. In some areas to lay down the fibre is almost impossible and impracticable like in downtown, hills and mountains. The effective cost of fibre optics is more than the wireless technology. The high speed and large bandwidth offered by light wave communications makes it a very attractive means of meeting the future demand for broadband internet access and HDTV broadcasting services. The table.1[4] depicts its upper hand over the other available technologies with regard to basic features like bandwidth, cost, installation time, security and maintenance. The RF technology is doing its wonders, but due to its high licensing cost and also cannot be scaled to the optical communication. The optimal solution offered by FSO in terms of meeting bandwidth requirements, scalability, less time to market and overall easy maintenance is the hallmark of this promising technology. Free space optics seem the most attractive solution, especially for short range communication like first and last mile connectivity[5]. The main resistance shown by FSO for long distance communication is due to the ill effects of weather on free space communication. Deployment of FSO is encouraged in the developed countries, but in developing countries, it is still a distant technology.

Table 1. Comparison of different bandwidth technologies. [4,16]

\begin{tabular}{|c|c|c|c|c|c|}
\hline Bandwidth Technology & $\begin{array}{l}\text { Twisted pair } \\
\text { cable }\end{array}$ & Coax cable & $\begin{array}{l}\text { Radio Frequency } \\
\text { (RF) wireless }\end{array}$ & Optical fibre & FSO \\
\hline Bandwidth & Low & High & Medium & Very High & Very High \\
\hline Cost & High & High & Very high & Very High & Low \\
\hline Security & Good & Average & Poor & Very Good & Very Good \\
\hline $\begin{array}{l}\text { Installation and maintenance } \\
\text { time }\end{array}$ & High & High & Medium & High & Low \\
\hline Reusability & Poor & Very poor & Low & $\begin{array}{l}\text { Sunk } \\
\text { technology }\end{array}$ & High \\
\hline Speed & Low & Moderate & Medium & Very high & High \\
\hline
\end{tabular}




\section{Need to go for Optical Wireless Communication}

The ever increasing demand for high data rate accordingly requires high bandwidth access system between end user and the backbone network. Presently twisted pair cable, coaxial cable and RF are commonly being used for this. This lack of availability of required bandwidth between the backbone network and the end user is known as the last mile connectivity bottleneck. The alternative solutions available to address this connectivity bottleneck problem are the use of optical fiber or free space optical links. . Optical fiber is the most obvious choice to address this problem. However, it is wired connectivity requires high cost and regular maintenance. Alternative Free-Space Optical communication (FSO) has become more and more interesting as an alternative to Radio Frequency (RF) communication over the last two decades. It is of paramount importance that the world is going to be digital in performing the day to day activities incoherent, coordinated, synchronized and at a faster rate. For example the government of India has launched a programme on August 21, 2014 to go to Digital India. The main objective of this programme is to make India digitally empowered by various sub-programs and transform the country into knowledge economy all across the globe. Here all the sectors are to be connected together in terms of sharing information, keeping track of ongoing activities and most importantly, making the access easier for every citizen of the country. The vision of the program is very evident that to transform the country from developing to the developed one. The mission of this umbrella programme is to convert the vision document into the action by connecting all the sectors of the nation, which includes small, medium and large sectors together and making the things public for absolute transparency and accountability, but without losing the sanctity of any institution or organization and without compromising the internal and external security climate of the nation. The two basic requirements to meet out this programme are connectivity and fast computing. For computing purpose very sophisticated high end processors of the state of art are available to meet out this requirement. But connectivity becomes the bottleneck, because of the high bandwidth requirement for communication purpose. After analyzing the available bandwidth technologies like twisted pair cable, coaxial cable, radio communication[6] and optical fiber, it is a crystal clear fact that without any doubt optical fiber is a mature technology till date to meet out the exponential demand of high bandwidth. The superiority of optical communication with reference to basic technological parameters compared to other means of communication, such as transfer of electric signals over copper is substantial[7]. Therefore, National Optical Fiber Network (NOFN) programme has been launched by government of India (GOI) to provide broadband highways by covering 250000 villages initially and followed by fiber to the home (FTH)[8]. Though the technology is wired and the implementation of NOFN and fibre to home (FTH) program are facing a number of challenges in terms of permission for digging, time to market, geographical problems, excessive cost, more human resource and breakdown of fiber etc, thereby hampering the actual target.

To address this problem there is a need to shift into optical wireless communication by keeping the basic principle of communication same and only replacing optical fiber communication by optical wireless communication like free space communication (FSO)[9]. FSO is a technology in which information is transmitted from one place to another place in the form of light without making use of the optical fiber cable. No doubt it has its own limitations like attenuation by atmospheric effects and the work is being done across the globe to minimize the ill effects of atmosphere on free space communication. But for a short distance communication, the FSO can be efficiently implemented and therefore it can be best alternative for first and last mile connectivity[10]. As per the report, about $93 \%$ businesses are within the range of one mile from the optical fiber network and cannot explore the full potential in terms of speed and bandwidth coming across the optical fiber due to non optical connection at the last and first end[7]. The radio links in place at the first and last mile connectivity can be easily replaced with free space optical links. So many experimental investigations have supported the concept of FSO connectivity in the communication networks. One of the experiment carried out on 10Gbits/s dual hop FSO setup in atmospheric turbulences for 100 to $500 \mathrm{~m}$ showed better BER performance and it also suggested the all optical switching overcomes the complexity of optical to electrical conversion[11]. One more investigation on 10Gbps data rate achieved over atmospheric turbulent channel at $10 \mathrm{~km}$ distance with NRZ, RZ and 
raised cosine modulation formats was carried out[12]. The performances were found acceptable, which was analyzed with eye diagram, BER and SNR. FSO has become more and more interesting as an alternative to RF communication over the last two decades. Ideally, FSO communication takes place at the speed of light.

\section{The peculiar advantages of FSO[7]}

- No permission required for spectral usage and hence no licensing fee needed as compared to the radio spectrum, therefore it is a cost effective technology and potentially they have low start-up and operational cost.

- The deployment time is very less along with easy maintenance.

- Virtually it offers unlimited bandwidth and hence very large capacity with high data rate transmission. They possess fiber like bandwidth.

- There are no optical interceptors/receivers to tap the optical beam from the link between an authentic pair of transceivers and therefore, very secure technology.

- They are flexible and easily deployable in multiple architectures, re-deployable, and environment friendly technology.

- There is no need to change the underlying transmission protocols and therefore, it is a transparent technology with better interoperability features.

- No cabling or intermediate transducers required and in ways small and light weight equipments.

- The beam divergence angle is very small and the power is focused on the target through a narrow beam of light and overall power consumption is less.

- The nature of signal is photonic, therefore less susceptible to electromagnetic interference and also offers electrical isolation.

- Impressive mean time before failure (MTBF).

- Offers flexible topology with the scalable character.

- The signal is highly focused on the target, almost negligible multipath propagation and therefore, less fading effects.

\section{Demerits [13]}

- Dispersive attenuation caused by fog, haze, snow, rain and dust particles.

- Atmospheric turbulence poses significant threat.

- Absorption and scattering degrades the overall performance.

- Physical obstructions can completely block the signal.

- The frequency selection is choosy keeping in view the eye safety.

- Building sways leads to pointing/alignment intolerances.

- Solar background radiation introduces a significant amount of noise especially when the signal beam comes parallel to sun rays.

\section{First and last mile (FLMC) Bottleneck Problem}

The first leg and final let to the telecommunication problem is first and last mile connectivity (FLMC). The broadband connectivity is getting poor in the developing countries like India. The reason behind this is excessive rush over the limited band and even paying more for the same. The problem is graven in the urban areas compared to rural areas and the growth of urbanization is also increasing. The telecommunication traffic load in the urban areas is many times more than in the rural areas and the possibility of digging and laying of optic fibre is difficult[14]. Keeping in view the concentration of high bandwidth demand, the radio links are coming short in the coming days and this is one of the reason to find out the another reliable medium at the end. To deliver the message at the beginning and receive at the end 
is the main concern. In the emerging markets like challenging locations, remote communities and highly busy cities, the operators are facing challenges in providing data bandwidth at the door steps. It is very difficult to connect the heavily urbanized communities with wired connectivity like FTH and therefore the viable alternative solution seems to be optical wireless link like FSO and it is of great importance[15].

Looking for ways to shorten the distance between the optical fibre and the user, fibre is going out from the network and is not reaching to the end user easily neither in congested nor in remote areas. The traditional approach of using copper/coax is likely on a verge of perish due to certain limitations with reference to current scenarios. The satellite option is unaffordable for most of the people, because of expensive service and no fixed performance. The pace of urban densification is going at high and data delivery essentially requires a medium compatible to speed with optic fibre for making a connection between backbone network, which is optic fiber and the end user.

\section{Proposed solution}

The dynamics of global networks have been changed with the advancement of optical technology. Free space optics (FSO) are the emerging solution to the first and last mile connectivity (FLMC). It is a line of sight communication and for that we have to establish a link between the end user and the core of networking. This is most effective for short range communications and can also be used to increase the distance using suitable relay assistance. A highly directed beam of light is used in this cable less optical communication technology. The infrastructure used at the transmitting and the receiving end must be installed correctly ensuring the maintenance of alignment and line of sight. Fig. 1 depicts the system using FSO links and it is evident that optical wireless technology is going to play a major role in the future global communications. The signal available at the source is converted from electrical to optical domain with the suitable optical source, either LED or laser and focused wirelessly to the receiving optical antenna, which in turn is connected to the optical fibre network at the back end and similarly at the last mile the signal coming from optical fibre is transmitted to the optical receiving antenna of the end user. The medium in between end user and the core network is an optical wireless channel (OWC) and the actual challenges of attenuation by the atmosphere are coming into play here. There are various mitigation techniques to counter the ill effects of the atmosphere like adaptive optics, spatial diversity, hybrid FSO/RF and relay assistance, etc [16]. In addition to these mitigation techniques, various innovative procedures involving suitable modulation methods have been proposed and experimented as well. The efficient coding pattern also enhances the level of security in FSO communication. With the exponential progress in optical wireless technologies, the FSO attracts the attention of global communication operators. In the communication networks of tomorrow, FSO is going to play a pivotal role and offering a workable solution to the explosive demand of bandwidth. The multifaceted approach is required in many sub-systems that balances the variables to produce the optimum mix. To support the idea of using FSO links to address the bottleneck problem by the scientific means has been taken into account in designing the solution. We know that about $90 \%$ of the end users fall at a distance of few kilometers (mostly 1 or $2 \mathrm{~km}$ ) away from the backbone network which is essentially an optical fiber core. The proposed solution is designed for maximum of $4 \mathrm{~km}$ under local weather conditions and the performance achieved are shall be suitable within the standard acceptable limits. Therefore, if we reduce the distance below $4 \mathrm{~km}$, the performance will further improve and is efficiently compatible to FLMC. The availability of optical bandwidth in a allows us to allocate separate frequency band to each user and hence we have selected optical frequency division multiplexing (OFDM) technique with WDM approach. 


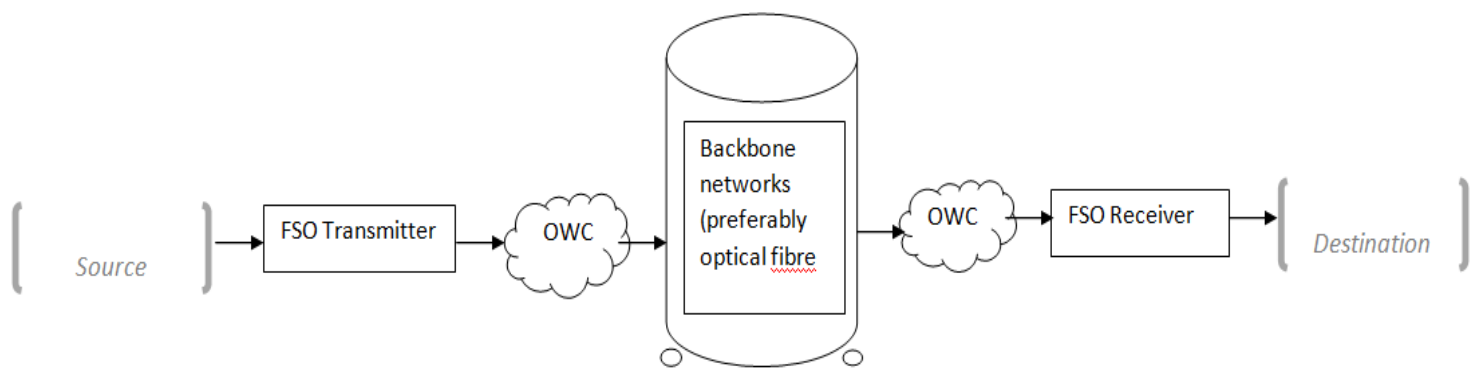

Fig. 1. Schematic diagram showing FSO link connecting the end user to the core network

\section{System Design}

The design taken into account supports the concept of using FSO communication at best to provide the efficient solution at first and last mile connectivity (FLMC). The design of a multichannel FSO system using NRZ modulation has been carried out in this investigation. The evaluation is done by adjusting the various parameters like data rate, beam divergence, transmitter power, receiver aperture and range. The system is designed at $40 \mathrm{~GB} / \mathrm{s}$ wavelength division multiplexing (WDM) at $4 \mathrm{~km}$ distance using a NRZ modulation with Avalanche Photo-detector (APD) and the results were analyzed on the basis of BER, QFactor and eye height.

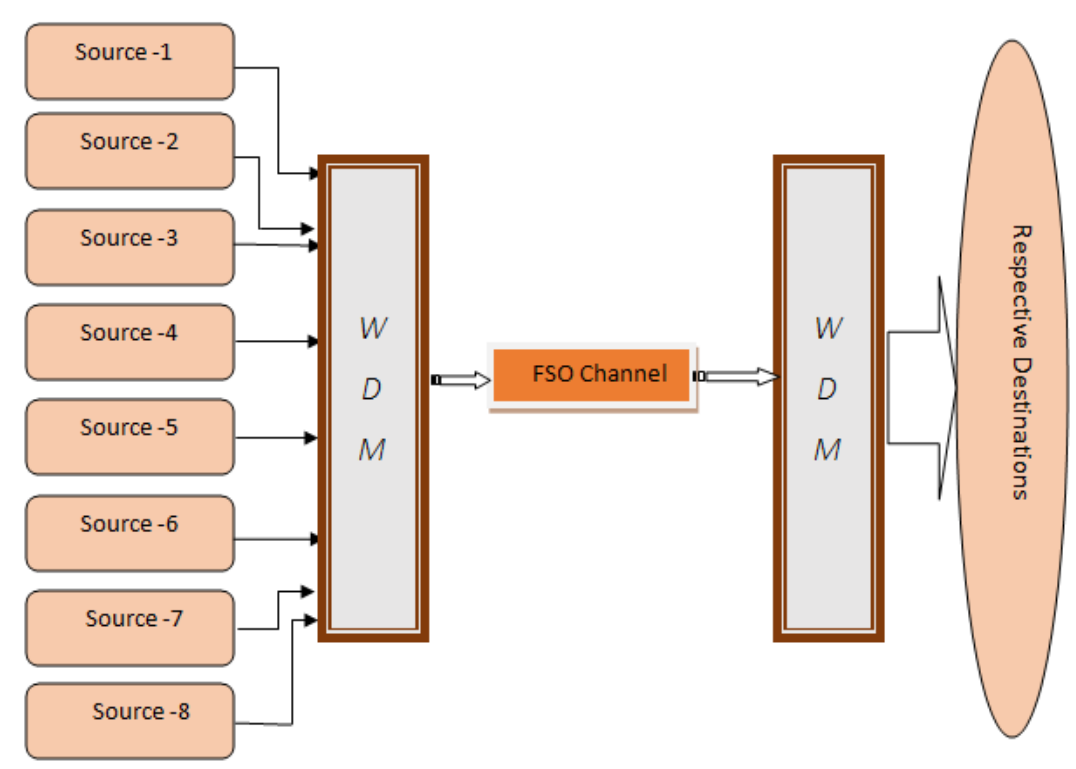

Fig.2. 8-channel WDM - FSO communication system simulation setup 


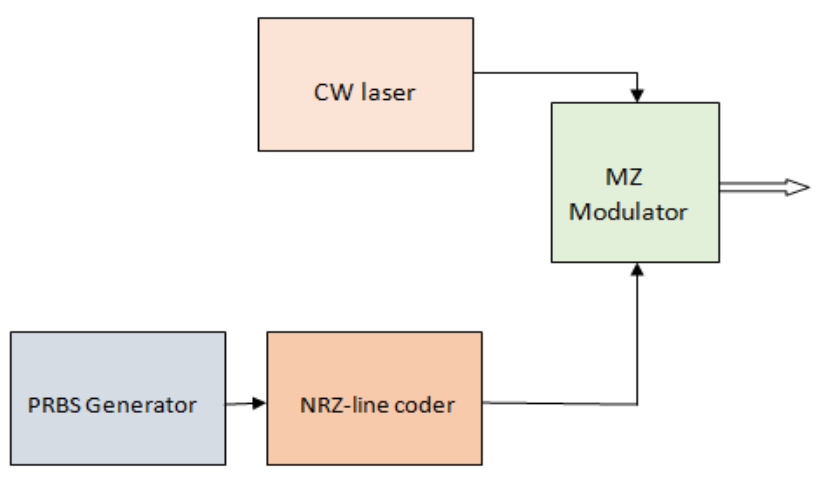

Fig.3. Transmitting side showing Optical Network Unit (ONU)

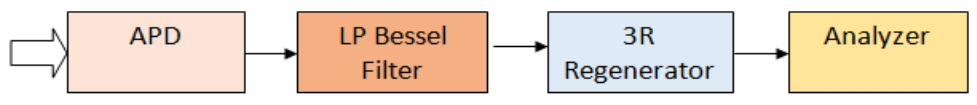

Fig.4. Receiving side showing Optical Network Unit (ONU).

The purpose of this experiment is to demonstrate a reliable communication through a free space optical channel based on varying turbulence approximation. In FSO communications, optical transceivers communicate directly through the air to form point to point line of sight links. The transmitter converts the electrical signal to an optical one, and sends it through the atmosphere (free space). The receiver converts the optical signal back to an electrical signal. The quality of the transmission line is characterized by the realized bit-error rate (BER). Figure 2 shows the simulation setup of the experiment. We have taken ' $n$ ' number of sources (where $n=1,2,3,4 \ldots 8$ ) and accordingly designed an FSO system using wavelength division multiplexing (WDM) with non return to zero (NRZ) line coding formats. The various Optical Line Terminal (OLT) components of WDM transmitter are shown in fig. 2.

The FSO system consists of transmitter, channel and receiver. The OLT comprises of a pseudorandom bit sequence (PRBS) generator, Non- Return-Zero (NRZ) line coder, continuous wave (CW) laser and MachZehnder modulator. This is the setup of each optical source and similarly we establish eight such sources each operating at different wavelength. A wavelength division multiplexing (WDM) transmitter transmits eight channels having channel spacing of $100 \mathrm{GHz}$ and each channel with input power 30mw transmitting $5 \mathrm{~GB} / \mathrm{s}$ data rate downstream.

An NRZ electric pulse generator is employed to provide the pulse shape for the digital data produced by the PRBS generator. The light signal generated by the CW laser and NRZ digital data is fed to the MZ modulator. The modulated optical signal from all the eight MZ modulators is inserted into the WDM unit. The multiplexing of all the eight inputs having different wavelengths is carried out by WDM multiplexor and the multiplexed output is finally propagated through the FSO channel to the destination terminal. An optical attenuator and noise adder is used to add attenuation with a certain level in $\mathrm{dB} / \mathrm{Km}$ into the underlying FSO channel. The input optical signal is attenuated with a particular level of attenuation through model attenuator. The desired level of optical attenuation is created anywhere in the topology by appropriate use of the model noise adder. The position of attenuator in the link range induces a variable level of distortion. If the position of attenuator is nearer to the transmitter, it will have more attenuation effect than positioning it near the receiver. The mechanism of directly manipulating the Amplified Spontaneous Emission (ASE) noise of an optical spectrum is provided by the noise model adder. The additional noise can be incorporated to the signal with varying profiles. The quality of the link is deteriorated by various attenuation factors leading to power loss. To compensate the power loss, the link margin needs to be employed with greater precision. The ratio of receiving power (Pr) and receiver threshold or sensitivity $(\mathrm{S})$ describes the Link Margin $\left(\mathrm{P}_{1}\right)$ and is usually expressed in $\mathrm{dB}$. 


$$
P_{l}=\frac{10 \log P_{r}}{S}
$$

We know that the average power of the signal should be more than the receiver sensitivity for efficient recovery at the receiver side. The sensitivity is usually given by the manufacturer and it ranges from -20 to $40 \mathrm{dBm}$. The power at receiver is expressed as:

$$
P_{r}=\frac{P_{t} e^{-\alpha l} A_{r x}}{(\theta l)^{2}}
$$

Where Pr and Pt are power at the receiver and transmitter respectively, Arx is a receiver aperture area, $\theta$ is divergence angle, $\alpha$ is atmospheric attenuation, and 1 is the distance between transmitter and receiver. The atmospheric attenuation coefficient is one of the important players, which sheds an effect on the overall performance of the system. It is best provided by Kim and Kruse model and is given by equation (3):

$$
\alpha=\frac{3.91}{V}\left(\frac{\lambda}{550 \mathrm{~nm}}\right)^{-q}
$$

Where ' $\lambda$ ' is the wavelength in $\mathrm{nm}$, ' $\mathrm{V}$ ' is the visibility in $\mathrm{Km}$ and scattering particle size distribution is denoted by ' $q$ '. Here we take the reference wavelength $550 \mathrm{~nm}$ as there is the maximum solar spectrum intensity. The model also provides the values of ' $\mathrm{q}$ ' and ' $\mathrm{V}$ ' in certain weather conditions and is given below table 2:

Table 2. The values of ' $\mathrm{q}$ ' and ' $\mathrm{V}$ ' at different weather conditions.

\begin{tabular}{rcll}
\hline S. No. & ' $\mathbf{q}$ ' & Length of visibility $(\mathbf{K m})$ & Visibility type \\
\hline 1. & 0.584 & $\mathrm{~V}<6.2$ & Low \\
2. & 1.29 & $6.2<\mathrm{V}<5.2$ & Average \\
3. & 1.59 & $\mathrm{~V}>5.2$ & Very high \\
\hline
\end{tabular}

As shown in equation (2), the power at the receiver is directly proportional to the transmit power and receiver aperture area, but inversely proportional to the link range and divergence angle. Exponential part of the equation is related to atmospheric attenuation and it has the strongest influence on the link quality. Adjusting the various parameters according to their relation to the received power, we attain the optimized receiver power. In this simulation work we also examined that performance is considerably improved by reducing the divergence angle. The LED or semiconductor lasers are the commonest devices used as transmitters at a wavelength of $800-850 \mathrm{~nm}$ or $1500-1550 \mathrm{~nm}$. The conditions and parameters taken into account are shown in table 3 .

The optical signals transmitted by the WDM transmitter with the help of triangular optical filter are received by the individual Optical Network Unit (ONU) at receiving side incorporated with the optical demultiplexer, which separates each channel with $100 \mathrm{GHz}(0.8 \mathrm{~nm})$ channel spacing. Figure 3 and Figure 4 shows the ONU components at transmitting and receiving side respectively. On receiving side the conversion of optical signal into electric signal is performed by using high sensitivity avalanche photo diode (APD). As we are dealing with higher data rate and the noise introduced by various means causes inter-symbol interference (ISI). We are making good use of BER tester to measure the error performance. 
Table 3. Various parameters taken into consideration.

\begin{tabular}{cll}
\hline S. No. & Parameters & Descriptions \\
\hline 1. & FSO link range & $4 \mathrm{KM}$ \\
2. & Number of channels & 8 \\
3. & Channel spacing & $100 \mathrm{GHz}$ \\
4. & Transmitter aperture diameter & $5 \mathrm{~cm}$ \\
5. & Receiver aperture diameter & $7.5 \mathrm{~cm}$ \\
6. & Beam divergence & $0.25 \mathrm{mrad}$ \\
7. & Attenuation & $3.5 \mathrm{~dB} / \mathrm{Km}$ \\
8. & Transmitter power & $30 \mathrm{~mW} / \mathrm{channel}$ \\
9. & Bit rate & $5 \mathrm{Gbps} / \mathrm{channel}$ \\
10. & Turbulence model & Gamma-Gamma \\
11. & Bessel filter order & 4 \\
12. & Cut off frequency of Bessel filter & Bit rate Hz*0.75 \\
\hline
\end{tabular}

\section{Results and Discussion}

With $100 \mathrm{GHz}$ channel spacing over FSO path, eight (8) channels are established by the WDM transmitter and the corresponding transmission wavelength spectrum is depicted in Fig.5.

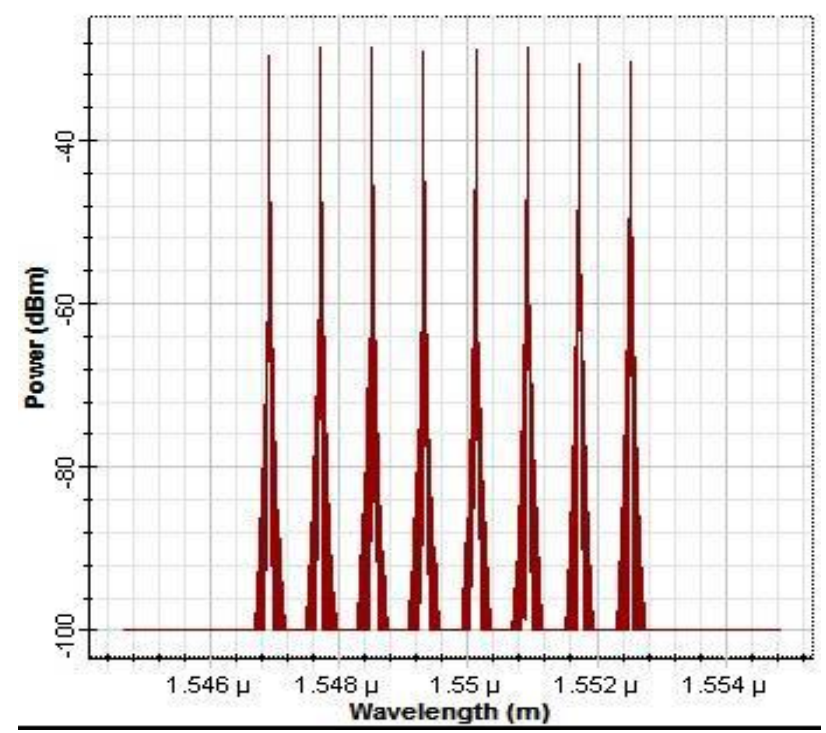

Fig.5. Output transmission wavelength spectrum of FSO channel.

Table 5. BER and Q-factor values for different channels for NRZ modulation format at $4 \mathrm{Km}$.

\begin{tabular}{|c|l|l|l|}
\hline S. No. & Wavelengths $(\mathbf{n m})$ & BER & Q-factor $(\mathbf{d B m})$ \\
\hline 1. & $\lambda 1=1552.52$ & $3.77 \mathrm{e}-21$ & 9.36 \\
\hline 2. & $\lambda 4=1550.11$ & $3.77 \mathrm{e}-20$ & 9.25 \\
\hline 3. & $\lambda 8=1546.91$ & $3.77 \mathrm{e}-25$ & 10.35 \\
\hline
\end{tabular}


We have taken three observations based on sampling method, $\lambda_{1}=1552.52, \lambda_{4}=1550.11$, and $\lambda_{8}=$ 1546.91 and it is evident that the reliable FSO communication has been achieved with much better performance. It is clear that with the increase in distance the BER also increases. Out of 8 channels, we are presenting the analysis based on three channels, which are channel number 1,4 , and 8 . The BER values of these channels with the NRZ modulation format at $1 \mathrm{Km}$ is $1.34 \mathrm{e}-288,1.97 \mathrm{e}-309$, and $7.83 \mathrm{e}-318$ respectively, and at $4 \mathrm{Km}$, the BER is $3.77 \mathrm{e}-21,1.05 \mathrm{e}-20$, and $2.02 \mathrm{e}-25$ respectively. Table 5 shows the BER and Q-factor values for the channel number 1, 4, and 8 for analysis and expected conclusion.

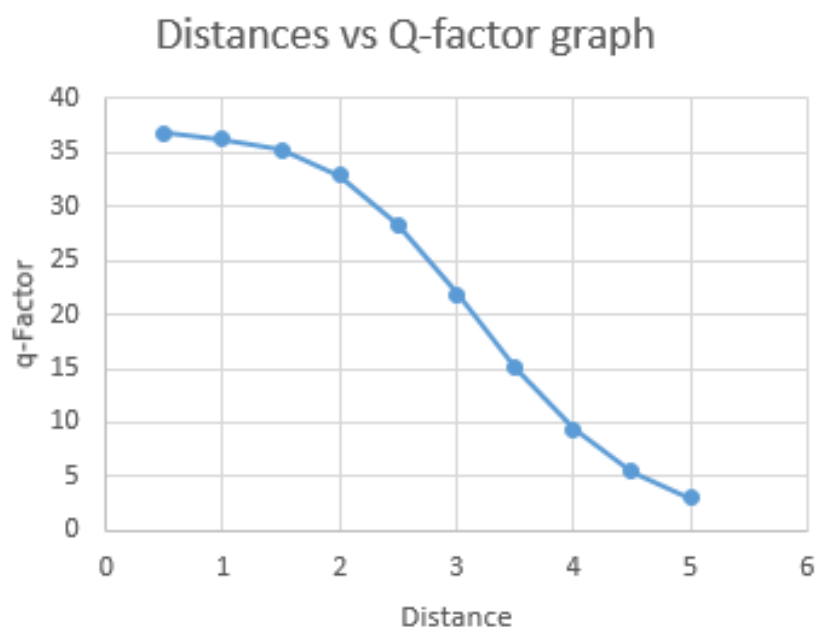

Fig.6. Distance and Q-factor relationship.

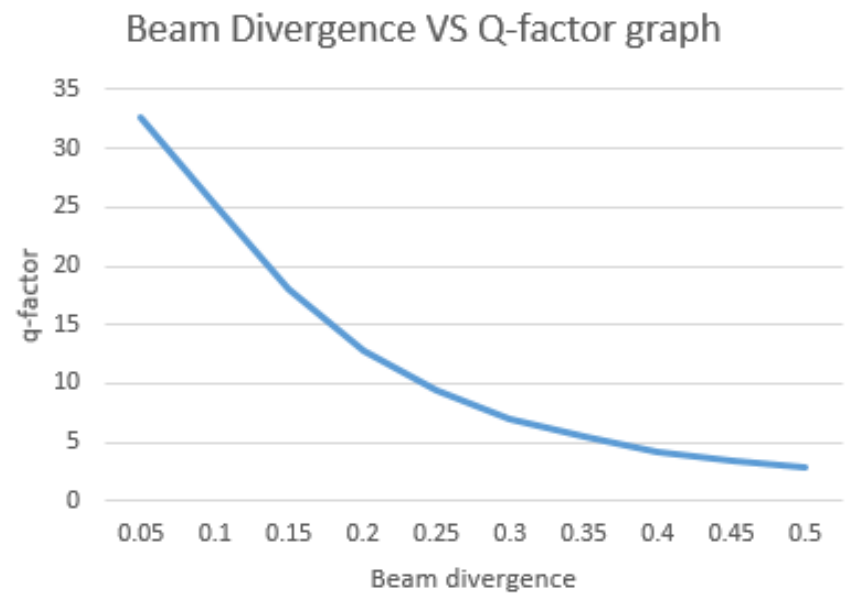

Fig.7. Beam Divergence and Q-factor relationship.

Fig.6. shows the relationship between the Q-factor and distance of $4 \mathrm{~km}$ with the NRZ modulation format of the FSO system under design consideration. For channels 1, 4, and 8 at $1 \mathrm{Km}$ distance, the Qvalues are $36.27,37.57$, and 38.8 and at $4 \mathrm{Km}$ the Q-values are 9.36, 9.25, and 10.35 respectively for the same channels. Therefore, it has been observed here also that distance also has an effect on the quality of receiving signal. If distance increases the quality of receiving signal decreases. Figure 7 compares system Q-factor at various beam divergences at $4 \mathrm{~km}$. For channel 8 the values of BER are $1.39 \mathrm{e}-72,3.97 \mathrm{e}-42$, $3 \mathrm{e}-15$, and $1.23 \mathrm{e}-2$ at $1.25 \mathrm{~GB} / \mathrm{s}, 2.5 \mathrm{~GB} / \mathrm{s}, 5 \mathrm{~GB} / \mathrm{s}$, and $10 \mathrm{~GB} / \mathrm{s}$ data rate. So it has been observed that 
when data rate per channel is increasing BER is also increasing. So there is a trade-off between data rate, distance, and BER.

The relationship between Q-factor and beam divergence is shown in Fig.7.

The eye diagram of data transmission with NRZ modulation through different channels at $4 \mathrm{Km}$ distance is shown in Fig. 8, 9 and 10. The eye opening with respect to its height and width of an eye diagram gives the idea of performance of data reception. The reception of the signal is much better if the eye opening is large and the small opening indicates the poor reception. The time interval over which received signal can be sampled without interference is defined by the width of an eye diagram. The performance of proposed WDM-FSO communication system using the NRZ modulation format is much better due to minimum inter-symbol interference (ISI), dispersion, and distortion as indicated by its large opening of eye diagram.

From the above such observations, it is clear that with the increase in the distance there is a decrease in performance. The results depict that FSO communication is best suitable for low range communications and hence clearly support the concept of using FSO links for first and last mile connectivity (FLMC) in the present and future generation networks. The results abstained reveals the concept of replacing radio links by optical wireless links without compromising with the quality of service. In addition of basic quality performance indicators, the data transmission at higher rate is achieved and hence the objective of the study undertaken is met.

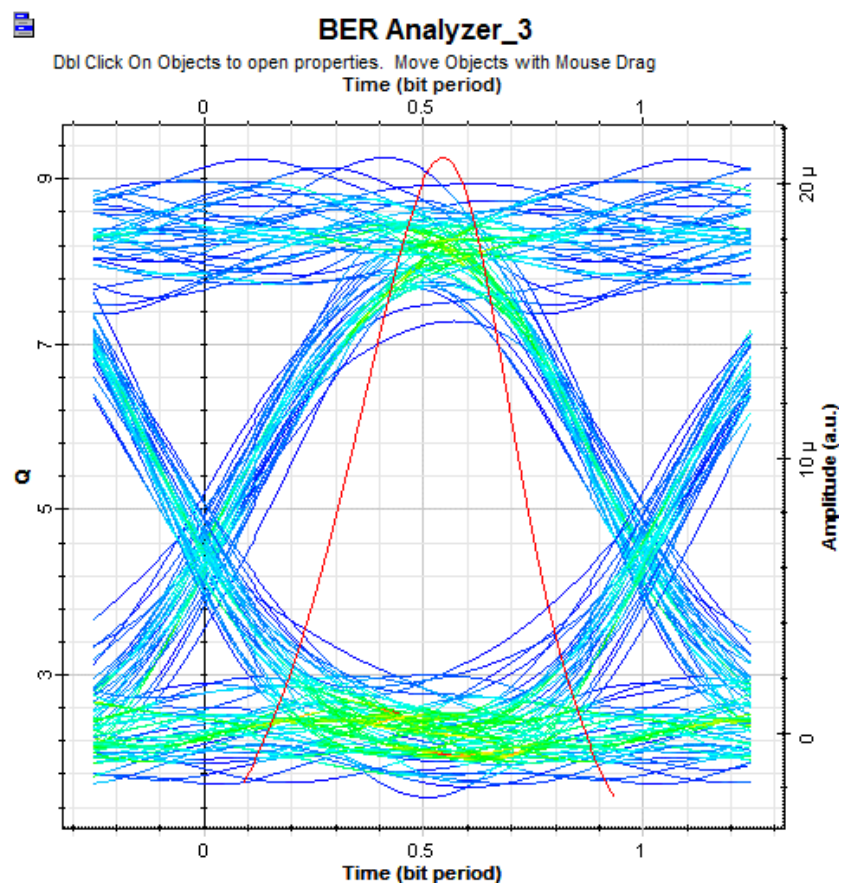

Fig.8. Eye Diagram at $\lambda_{1}$ 
Free Space Optics (FSO): A Promising Solution to First and Last Mile Connectivity (FLMC) in the Communication Networks

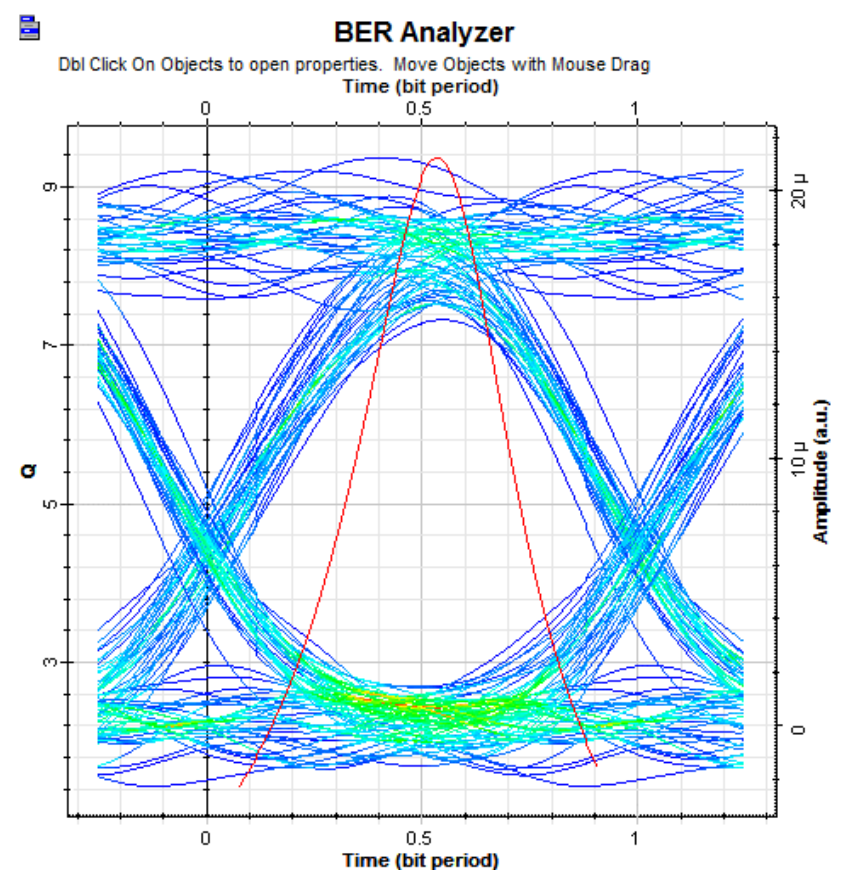

Fig.9. Eye Diagram at $\lambda_{4}$

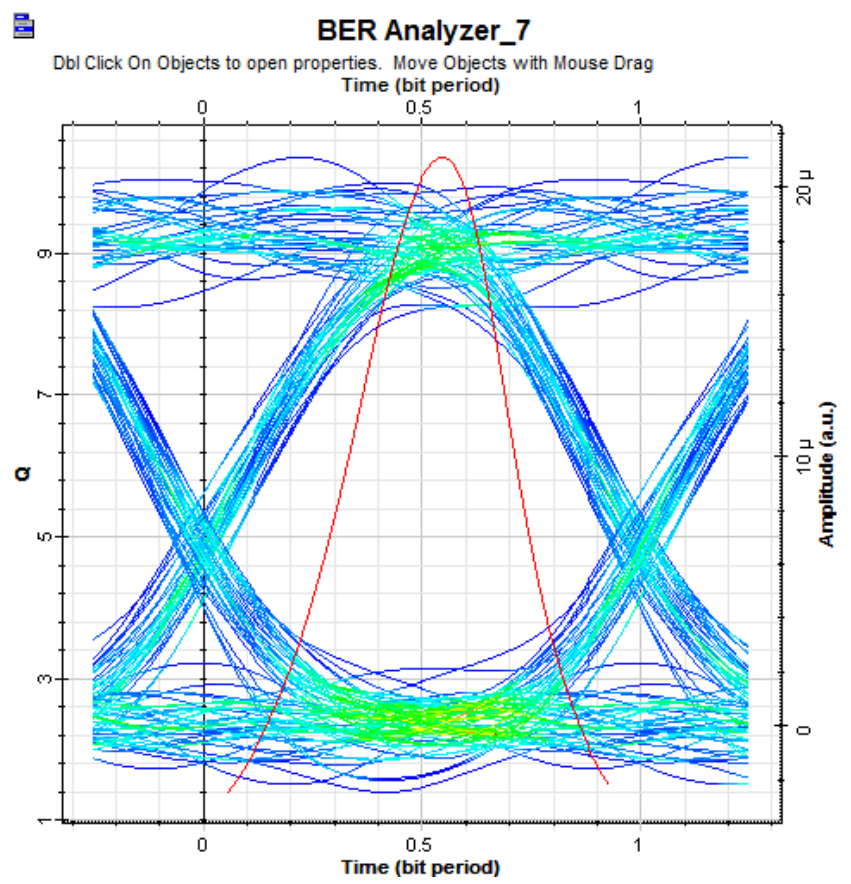

Fig.10. Eye Diagram at $\lambda 8$ 


\section{Applications [5]}

- Extension in the telecommunication networks

- Last mile connectivity

- First mile connectivity

- LAN to LAN connectivity

- Storage area networks

- Intra campus connections

- Fibre compliment

- Access technology

- Backhaul

- Building to building connectivity

- $\quad$ Ship to ship

- Aircraft to ground

- Satellite to ground

- Point to multipoint connectivity

- Metro network extensions

- Simplifying network switching (no more O-E-O conversion required)

- Military applications (Due to excellent security feature)

- Outdoor wireless access

- Used to bypass local loop systems

- Fibre backup

- Service acceleration

- Can be used in SONET rings

\section{Conclusion}

The problem of first and last mile connectivity can be substantially addressed by FSO link and its necessary infrastructure could be installed in a short time. This will essentially reduce the bottleneck problem between the broadband backbone network and the end user. FSO plays a major role in going for all optic communication systems at the present time and can bridge the last and first mile connectivity in the communication networks. It will definitely unclog the bottleneck between end user and optical fibre backbone. It is evident from the said study that FSO can meet out all essential requirements in terms of speed, capacity, security and quality provided the ill effects of atmospheric turbulence are addressed. FSO transmission systems can facilitate fast data rates in the communication networks. The said work undertaken is justified, where the WDM-NRZ modulation over FSO links gives the performance with regard to Q-factor and BER as acceptable for reliable communication. We know internet connectivity over optics is considered to represent the future of broadband connectivity. Today, the transfer of businesses moving servers to the cloud and the evolution of big data demands rapidly uploads and downloads than ever before. The work undertaken can be extended to address the problem of bandwidth bottleneck. The results clearly indicate that FSO links are the variable approach for addressing emerging FLMC broadband bottlenecks.

\section{References}

[1] H. Henniger and O. Wilfert, "An introduction to free-space optical communications," Radio Eng., vol. 19, no. 2, pp. 203-212, 2010.

[2] Z. Wang, W. Zhong, C. Yu, and S. Fu, "Performance improvement of on-off-keying free-space optical transmission systems by a co-propagating reference continuous wave light," vol. 20, no. 8, pp. 9284-9295, 2012.

[3] M. Leeson, "Channel Models for Optical Wireless Communication Systems," no. June 2009, 2016.

[4] A. Malik and P. Singh, "Free Space Optics : Current Applications and Future Challenges," vol. 2015, no. c, 2015. 
[5] A. V. L. Solution and V. Ramasarma, "Free Space Optics :," no. December, pp. 22-30, 2002.

[6] R. A. Alsemmeari, S. T. Bakhsh, and H. Alsemmeari, "Free Space Optics Vs Radio Frequency Wireless Communication,” Int. J. Inf. Technol. Comput. Sci., vol. 8, no. 9, pp. 1-8, 2016.

[7] H. Willebrand and B. S. Ghuman, "Free-Space Optics : Enabling Optical Connectivity in Today' s Networks."

[8] H. F. Hanafi, C. S. Said, and M. H. Wahab, "Improving Students ' Motivation in Learning ICT Course With the Use of A Mobile Augmented Reality Learning Environment Improving Students ' Motivation i n Learning ICT Course With the Use of A Mobile Augmented Reality Learning Environment," 2017.

[9] J. Akella, "Multi-channel Communication in Free-Space Optical Networks for the Last-mile."

[10] S. Mughal, R. Shah, Z. Hussain, and Z. A. Bhutto, "A Dominant Last Mile Connectivity Solution for 3G / 4G," vol. 1, no. 1, pp. 23-27, 2017.

[11] J. Libich, M. Komanec, S. Zvanovec, P. Pesek, W. O. Popoola, and Z. Ghassemlooy, "Experimental verification of an all-optical optics link under turbulence regimes," vol. 40, no. 3, pp. 10-13, 2015.

[12] S. Chaudhary, "Implementation of FSO Network under the Impact of Atmospheric Turbulences," vol. 75, no. 1, pp. 34-38, 2013.

[13] N. Kumar and A. K. Rana, "Impact of various parameters on the performance of free space optics communication system," Optik (Stuttg)., vol. 124, no. 22, pp. 5774-5776, 2013.

[14] M. Torlak, "Optical Fibers."

[15] H. Zhou, S. Mao, and P. Agrawal, "Optical power allocation for adaptive transmissions in wavelength-division multiplexing free space optical networks,” Digit. Commun. Networks, vol. 1, no. 3, pp. 171-180, 2015.

[16] H. Kaushal, G. Kaddoum, and C. Engineering, "Free Space Optical Communication : Challenges and Mitigation Techniques," pp. 1-28.

\section{Authors' Profiles}

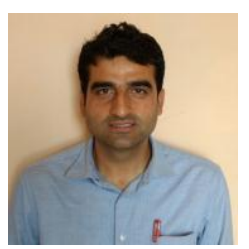

M Mubasher Hassan is pursuing Ph.D from Department of ECE National Institute of Technology (NIT) Srinagar, J\&K, India. He is working on performance improvement in FSO communication for next generation networks. He has received his M.Tech degree from the NIT Srinagar in 2007 and presently working as Assistant Professor at BGSB University Rajouri J\&K, India. He has received his B.E degree from the Jammu University in 2004. mubasher2003@gmail.com

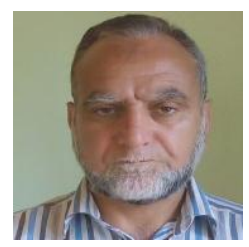

G M Rather has received his Ph.D degree from I.I.Sc Banglore. He is presently working as Professor in the Department of ECE, National Institute of Technology (NIT) Srinagar, J\&K, India. He has a vast experience of teaching and research, particularly in the field of communication systems, computer networks and advanced wireless systems. gulammohdrather@yahoo.co.in

How to cite this paper: M Mubasher Hassan, G M Rather, "Free Space Optics (FSO): A Promising Solution to First and Last Mile Connectivity (FLMC) in the Communication Networks", International Journal of Wireless and Microwave Technologies(IJWMT), Vol.10, No.4, pp. 1-15, 2020.DOI: 10.5815/ijwmt.2020.04.01 